\title{
Automated Screening of MRI Brain Scanning Using Grey Level Statistics
}

\author{
Ali. M. Hasan and Farid Meziane
}

\begin{abstract}
This paper describes the development of an algorithm for detecting and classifying MRI brain slices into normal and abnormal by relying on prior-knowledge, that the two hemispheres of a healthy brain have approximately a bilateral symmetry. We use the modified grey level co-occurrence matrix method to analyze and measure asymmetry between the two brain hemispheres. 21 co-occurrence statistics are used to discriminate the images. The experimental results demonstrate the efficacy of our proposed algorithm in detecting brain abnormality with high accuracy and low computational time. The dataset used in the experiment comprises 165 patients with 88 patients having different brain abnormalities whilst the remainder do not exhibit any detectable pathology. The algorithm was tested using a ten-fold cross-validation technique with 100 repetitions to avoid the result depending on the sample order. The maximum accuracy achieved for the brain tumors detection was 97.8\% using a Multi-Layer Perceptron Neural Network.
\end{abstract}

Index Terms-Magnetic resonance imaging, Modified Grey Level Co-occurrence Matrix, Principle component analysis, Multi-layer Perceptron Neural Network, Support Vector Machine, Linear Discriminant Analysis.

\section{Introduction}

Medical image processing has expanded over the last decade attracting researchers with expertise from different fields such as mathematics, computer sciences, engineering, biology and medicine. The medical image processing field has become very important and its applications in clinical practice and biomedical imaging help to examine and diagnose human patients.

Brain tumours and stroke lesions are considered to be crucial cases in medical imaging as their accurate detection and segmentation have a significant influence on clinical diagnosis, predicting prognosis and treatment in addition to being beneficial for the general modelling of the brain's pathologies and its anatomical construction [1]. The detailed information collected from these images can also be used to index large databases of the archived images of brain tumors for various studies and purposes. Historical data can then help clinicians and radiologists to diagnose and treat new patients by determining the effectiveness of various medicine and procedures on previous patients with similar tumor volume and tumor area [2].

In current clinical routine, clinicians spend an increasing part of their time diagnosing and interpreting medical images. They need to have a high level of experience to carry out manual and accurate dilineation and classification of these images if they are to be used in the final diagnosis. However, due to the large number of slices which are produced by medical scanners, the manual detecion of the tumors is considered a very cumbersome time consuming task and prone to human errors. They are evaluated either based on qualitative criteria by observing hyperintense tissue appearance or by depending on primitive quantitative measures such as the largest diameter visible from axial viewing [3, 4].

The Iraqi Ministry of Health reported that the average annual number of registered cancerous brain tumor cases has increased significantly since 1990 due to decades of wars, sanctions and occupation [5]. This study was conducted in collaboration with the MRI Unit of the Al-Kadhimiya Teaching Hospital in Iraq which faced many problems in diagnosing and issuing diagnostic reports for the large number of inpatient and outpatient cases. Indeed, the average number of patients received daily by the MRI unit is over 110 patients for a six working days week totalling about 2640 patients scanned monthly. Therefore, developing an automated system for brain tumor detection is beneficial and desirable to reduce both human errors and workload in the MRI units in Iraq.

Typical medical imaging techniques include ultrasonography, computed tomography (CT) and magnetic resonance imaging (MRI). Among these technologies, MRI is an excellent medical imaging technique for studying the brain and it is widely used due to its ability to differentiate soft tissues with a significant spatial resolution that reached a $1 \times 1 \times 1 \mathrm{~mm}$ voxel size. It is more sensitive to local changes in tissue water since these changes reflect the physiologic alternation that can be detected and visualized by MRI. Furthermore, it is a non-invasive technology and is different from other technologies because of its ability to generate multiple images of the same tissue with different contrast visualization and different image acquisition protocols. These multiple MRI images provide additional useful anatomical information to help the clinicians study the brain pathology more

Ali. M. Hasan is the corresponding author: Address: School of Computing, Science and Engineering, University of Salford, M5 4WT, UK. Emails: ali11alwaeli@yahoo.com, a.hasan4@edu.salford.ac.uk.

Farid Meziane. Address: School of Computing, Science and Engineering, University of Salford, M5 4WT, UK. Email: f.meziane@ salford.ac.uk. 
precisely. Indeed, quantitative analysis of MRI brain scans to acquire knowledge about the human brain structure has increased dramatically in recent years because of a variety of diseases that can alter the brain structure. By analyzing these alternations, it is possible to understand clearly these diseases and potentially diagnose them quickly and accurately $[1,3]$.

The main objective of this study is to develop and evaluate an automated screening algorithm of MRI brain scanning images to discriminate normal and pathological patients without any clinician's interception; hence reducing clinicians' data examination and interpretation time. The remainder of the paper is organized as follows, in Section 2, we review some related work and introduce the contribution of this research. In Section 3, material and methods are described. In sections 4 and 5 we demonstrate how the features are extracted and classified. The experimental results are discussed in Section 6. Finally, the conclusions are drawn in Section 7.

\section{Related Work}

Kharrat, et al. [6] used Grey Level Co-occurrence Matrix ( GLCM) and wavelet features to recognize and classify MRI brain scanning images into normal, benign and malignant. The most relevant features were selected using genetic algorithms (GAs) and the classification was carried out using a Support Vector Machine (SVM). 97\% accuracy was achieved in classifying a dataset of 83 MRI brain images. Pantelis [7] developed a medical system to classify and discriminate the normality and abnormality of MRI brain scanning images by combining three approaches for texture features; GLCM, first order statistical method and Grey Level Run Length Matrix (GLRLM) and the dimensionality of the extracted features were reduced by using the Wilcoxon non-parametric test which is a non-parametric statistical hypothesis test used when comparing two related samples. The most relevant features were retained when the P-value, a function of the observed sample results, is less than 0.001. SVM was used to classify a dataset of 67 patients and the maximum classification accuracy obtained was 93\%. Lahmiri and Boukadoum [8] developed a new methodology for automatic features extraction from biomedical images using wavelet features and the Gabor filter with different frequencies and spatial orientations. The classification was performed using SVM and accuracies of $86 \%, 68 \%$ and $50 \%$ were achieved on MRI brain images, mammograms and retina respectively. Similarly, Sachdeva, et al. [9] proposed a multiclass brain tumor classification algorithm by using various techniques for feature extraction; Laplacian of Gaussian (LoG), GLCM, Rotation Invariant Local Binary Patterns (RILBP), Intensity-Based Features (IBF), and Directional Gabor Texture Features (DGTF). Finally these features were classified using Artificial Neural Network (ANN) after implementing Principle Component Analysis (PCA) for data reduction. An overall classification accuracy of $91 \%$ was achieved to classify a dataset of 428 MRI brain scanning images. Nabizadeh and Kubat [10] proposed a fully automated algorithm by using five effective texture-based statistical feature extraction methods; first order statistical features, GLCM, GLRLM, histogram of oriented gradient HOG and linear binary pattern (LBP). PCA was used for feature dimension reduction and 97.4\% accuracy was achieved for classifying the MRI brain scans of 25 patients by using SVM. Ruppert, et al. [11] proposed an algorithm for extracting the mid-sagittal plane (MSP) by searching the plane that maximizes a bilateral symmetry measure based on extracting the edge features from MRI brain image, and then measuring the similarity using the correlation between the left and right sides of an edge image with respect to a candidate cutting plane. Jayasuriya and Liew [12] proposed an automated algorithm for detecting the MSP of the brain by exploiting the property that the longitudinal fissure in T1 weighted MRI images appears as a dark area. Nabizadeh and Kubat [10] separated the brain into two hemispheres by finding the longest diameter that represents the MSP of the brain. Since most machine learning algorithms can suffer from both and excessive number of predictors and they are designed to use the most appropriate predictors when making their decisions. The most appropriate predictors denote the most promising predictors that are used to split the given data into classes with more discriminating power. Therefore the predictor selection and dimension reduction become very popular and widely studied [13].

The majority of the proposed approaches in the field of brain abnormalities detection and segmentation lack full automation and depend on multi-modalities of MRI data due to the variability and complexity of the location, size, shape and texture of the lesions in addition to the similarity of the intensity between brain lesions and normal tissues. We address the above-mentioned shortcomings by proposing a new algorithm that is:

1-Independent of atlas registration in order to avoid any inaccurate registration process that affects the precision measurement of the tumors' classification.

2-Fully automatic algorithm with no need for any human intervention or initialization.

3-Using only one statistical method for texture features extraction to detect the normality and abnormality of a MRI brain.

\section{Material and Methods}

The main objective of this research is to develop and evaluate an automated screening algorithm for detecting and classifying MRI brain scans into normal or abnormal within a short time. The overall flow chart of the proposed algorithm is shown in Fig. 1. It starts with the data collection phase from the Iraqi hospital, a set of algorithms in the pre-processing stage will then be implemented and they are followed by a features extraction algorithm and classification. 


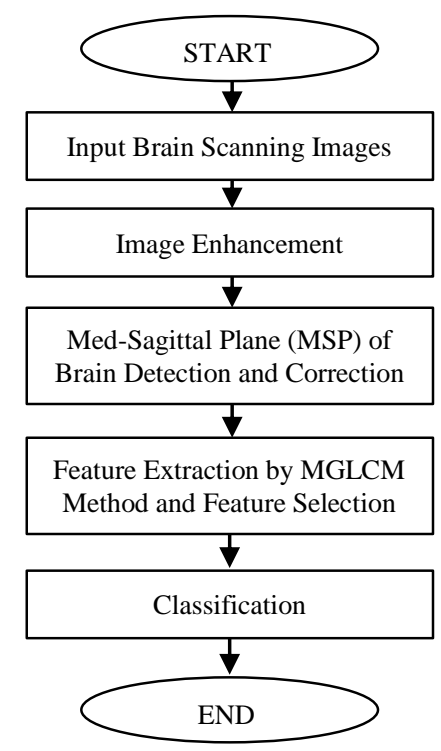

Figure 1: Flow chart of the proposed algorithm.

\subsection{Data Collection}

Data collection is an important step in this study. T2 weighted images in axial viewing of 165 patients were collected from the MRI Unit of Al-Kadhimiya Teaching Hospital in Iraq. Each patient has 32 slices with a slice resolution of (432×512 pixels). T2 weighted modality is used in this study for detecting brain tumors because most of the brain tumors appear hyperintense relative to normal brain tissue [14]. The dataset was collected using a SIEMENS MAGNETOM Avanto 1.5 Tesla scanner and PHILIPS Achieva 1.5 Tesla scanner. The provided dataset consists of tumors with different sizes, shapes, locations, orientations and types.

\subsection{Image Preprocessing}

The preprocessing step includes implementing three main algorithms on the slices of the MRI brain scans as preparation for the features extraction step. $t$ includes resizing the dimensions of MRI slices, image enhancement by the Gaussian filter and MRI intensity normalization - necessary because of intra-scan and inter-scan image intensity variations $[1,10,15]$.

\subsection{Mid- Sagittal Plane Detection and Correction}

MSP identification is an important initial step in brain image analysis as it provides an initial estimation of the brain's pathology assessment and tumor detection [12]. The human brain is divided into two hemispheres that have approximately bilateral symmetry around the MSP. This means that most of the structures in one side of the brain have a counterpart on the other side with similar shape and location. The two hemispheres are separated by the longitudinal fissure that represents a membrane between the left and right hemispheres [11]. The MSP extraction methods can be divided into two groups [11, 16] Content-based methods that are based on finding a plane that maximizes a symmetry measure between both sides of the brain [11] and Shape-based methods that use the inter-hemispheric fissure as a simple landmark to extract and detect the MSP [16].

In this study, we are interested in determining the orientation of the patient's head instead of depending on measuring the symmetry to identify the brain MSP as we are using the Principal Components Analysis (PCA) method to compute the distinctive principle axes that are orthogonal to each other. Those axes are used to characterize the patient's head by representing the spatial distribution of the mass [16]. The proposed algorithm includes five steps. The first step separates the brain from the background by using the histogram thresholding approach because the background normally has a much higher number of unavailing pixels [1]. The second step also uses the holes filling morphological operator to fill the holes that are defined as a background region of a binary image and surrounded by connected borders of foreground [17] as shown in Fig. 2. 


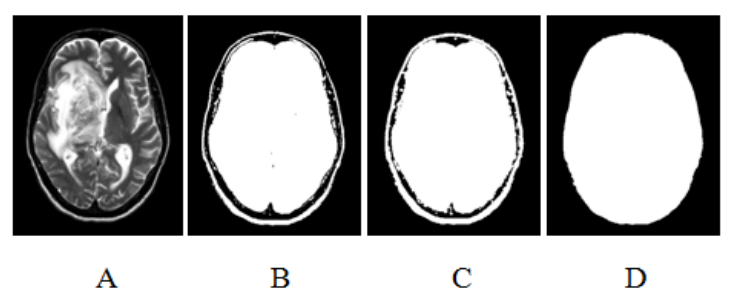

Figure 2: An example for MRI brain scanning image segmentation, A) Original MRI image,

B) Segmented MRI image with threshold equal to 25, C) Dilated MRI image, and

D) Filled holes image.

The third step determines the orientation of the patient's head using PCA. The PCA method essentially attempts to transfer the coordinates of the original data to a new coordinates system such that the maximum variation in the data comes to lie on the first coordinate. This is known as the first principal component. The second maximum variation in the data lies on the second coordinate and so on [17]. The new coordinates of the given data are estimated by calculating the eigenvectors which point in the direction of the new dataset coordinates. The desirable coordinate that has the highest eigenvalues, passing through the maximum variation of data, represent the orientation of the patient's head [17]. The angle $\theta$ between the X-axis and X'-axis represent the degree of skewness of the patient's head during the MRI test as shown in Fig. 3 and is calculated using Equation 1.

$$
\theta=\tan ^{-1} \frac{V_{2}}{V_{1}}
$$

1

Where, $V_{1}$ and $V_{2}$ are the eigenvectors which are related to the maximum eigenvalues.

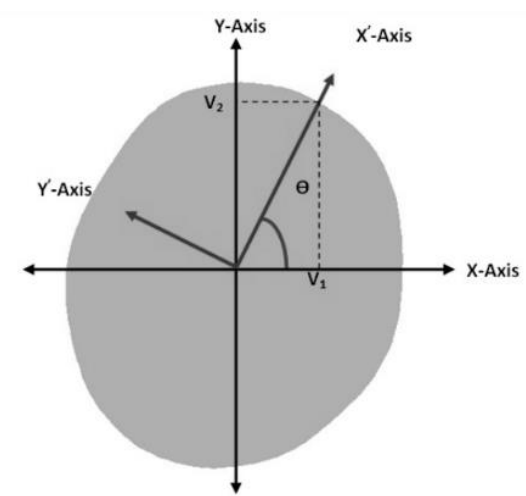

Figure 3: Original and new coordinates of a patient's head.

The fourth step is a Geometrical transformation which is widely used in computer graphic and image analysis. It includes two basic steps. First, the pixel coordinates transformation and second, the brightness interpolation [17]. In this study, it is used to rotate and correct the patient's head after the degree of wobbling is calculated in the previous step. The fifth step is the positioning of the patient's head in the centre of the MRI image because identifying the brain's abnormality depends essentially on measuring the symmetry between the two brain's hemispheres.

The MRI brain slices of each patient have the same degree of skewness; therefore the MSP detection and correction algorithm is implemented on a single slice instead of using all slices to avoid computational complexity. The preferable slice for implementing the MSP detection and correction algorithm is the slice which is located in the middle of all the slices. The MRI brain scanning image is divided evenly into two halves, each half containing one brain hemisphere. Hence after correcting and rotating the patient's head, it is easy to position the brain's MSP exactly in the middle of the MRI scanning image by shifting the patient's head either left or right. Eventually, the brain image is centralized and skewness free.

\section{Proposed Feature Extraction Method}

The choice of appropriate features depends on the particular image and application. The extracted features should be reliable i.e. the features of the same class should have similar values that are uncorrelated in order to avoid computation waste [1], and they should be extracted in a reliable way $[37,38]$. In this study, we only consider the textural features because there is no colour information or regular shapes of the tumors that could be extracted. The texture features will be extracted from MRI brain scanning images to encode clinically valuable information using the proposed modified grey level co-occurrence matrix 
(MGLCM) method. The texture features will also be used to measure statistically the similarity between the two separated hemispheres of the brain. A prior preprocessing algorithms that should be taken to prepare the MRI brain scanning image for texture features extraction by the proposed method is used.

\subsection{MRI Brain Scanning Image Preparing}

This step includes the implementation of a set of image preprocessing algorithms to prepare and make the MRI brain scanning images more appropriate for implementing the MGLCM method. The input for this step is a corrected MRI brain scanning image, and the output image includes only the patient's head with dimensions of $(512 \times 512)$ pixels. Initially, the MRI image is cropped from the upper margin of the image to the upper boundary of the skull. The same procedure is then used done for cropping the MRI image from the bottom margin of the image to the bottom boundary of the skull. The left and right boundaries can then be identified by measuring the distance between the upper and bottom boundaries such that, the left and right boundaries are away from the middle of the MRI image which denotes the MSP of the brain, by a half distance between the top and bottom boundaries as shown in Fig. 4. Finally, every cropped MRI brain scan image is resized to $(512 \times 512)$ pixels before implementing the MGLCM method.

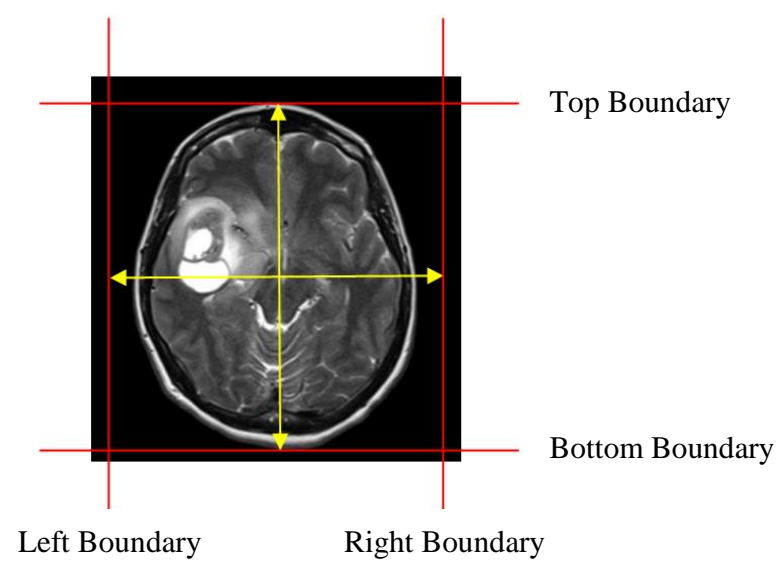

Figure 4: MRI brain scanning image cropping.

\subsection{Modified Grey Level Co-occurrence Matrix MGLCM Algorithm}

MGLCM is a second order statistical method. It is proposed in this study to generate texture features of MRI brain scans by computing the joint frequencies of all pairwise combinations of the grey levels configuration of each pixel in the left hemisphere, which is considered as a reference pixel, with one of the nine opposite pixels in the right hemisphere according to the nine offsets $\theta=(45,45),(0,45),(315,45),(45,0),(0,0),(315,0),(45,315),(0,315),(315,315)$, and one distance $d=1$, as shown in Fig. 5. Subsequently, because each pixel on the left hemisphere has nine opposite pixels on the right hemisphere, nine co-occurrence matrices are obtained for each MRI brain scanning image.

Thereafter, each co-occurrence matrix is normalized by the sum of all its elements to calculate the co-occurrence relative frequency between the grey levels of joint pixels in the brain hemispheres. The nine co-occurrence matrices are defined using Equation 2.

$$
P(i, j)_{\left(\theta_{1}, \theta_{2}\right)}=\frac{1}{256^{2}} \sum_{x=1}^{512} \sum_{y=1}^{256}\left\{\begin{array}{l}
1, \text { if } L(x, y)=i \text { and } R(x+\Delta x, y+\Delta y)=j \\
0, \text { Otherwise }
\end{array}\right.
$$




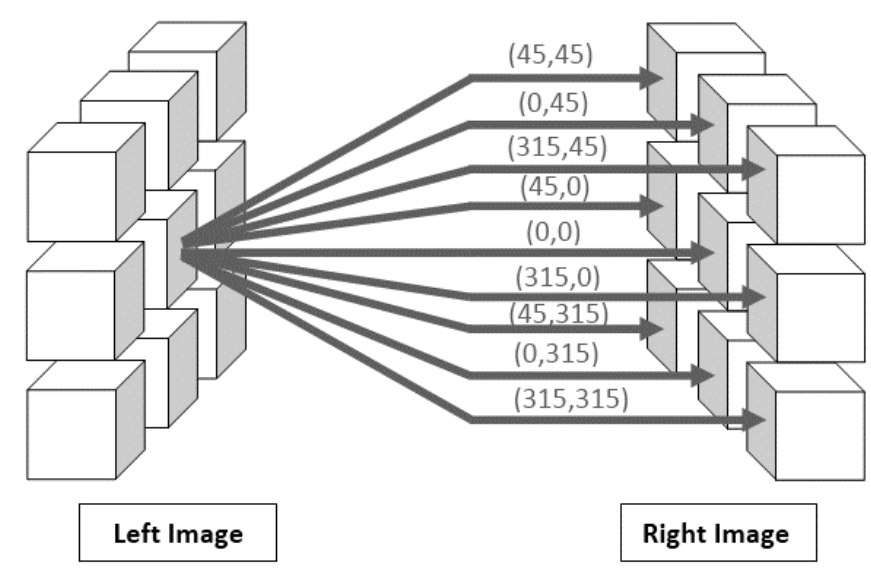

Figure 5: How a reference pixel relates with its opposite nine pixels.

Where $\mathrm{L}$ and $\mathrm{R}$ denote the left and right hemispheres respectively and both of them have a size of $(512 \times 256)$ pixels. $\mathrm{P}$ is the resultant co-occurrence matrix. $\Delta \mathrm{x}$ and $\Delta \mathrm{y}$ are changed dependent upon the directions of the measured matrix with the following rules used:

$$
\begin{aligned}
& \text { if } \theta_{1}=0 \text { and } \theta_{2}=0 \text { then } \Delta \mathrm{x}=0 \text { and } \Delta \mathrm{y}=0, \\
& \text { if } \theta_{1}=0 \text { and } \theta_{2}=45 \text { then } \Delta \mathrm{x}=-1 \text { and } \Delta \mathrm{y}=0, \\
& \text { if } \theta_{1}=0 \text { and } \theta_{2}=315 \text { then } \Delta \mathrm{x}=1 \text { and } \Delta \mathrm{y}=0, \\
& \text { if } \theta_{1}=45 \text { and } \theta_{2}=0 \text { then } \Delta \mathrm{x}=0 \text { and } \Delta \mathrm{y}=1, \\
& \text { if } \theta_{1}=315 \text { and } \theta_{2}=0 \text { then } \Delta \mathrm{x}=0 \text { and } \Delta \mathrm{y}=-1, \\
& \text { if } \theta_{1}=45 \text { and } \theta_{2}=45 \text { then } \Delta \mathrm{x}=-1 \text { and } \Delta \mathrm{y}=+1, \\
& \text { if } \theta_{1}=315 \text { and } \theta_{2}=45 \text { then } \Delta \mathrm{x}=-1 \text { and } \Delta \mathrm{y}=-1, \\
& \text { if } \theta_{1}=315 \text { and } \theta_{2}=45 \text { then } \Delta \mathrm{x}=-1 \text { and } \Delta \mathrm{y}=-1, \\
& \text { if } \theta_{1}=315 \text { and } \theta_{2}=315 \text { then } \Delta \mathrm{x}=1 \text { and } \Delta \mathrm{y}=-1, \\
& \text { if } \theta_{1}=45 \text { and } \theta_{2}=315 \text { then } \Delta \mathrm{x}=1 \text { and } \Delta \mathrm{y}=1 .
\end{aligned}
$$

The resultant co-occurrence matrices are approximately symmetric around the forward diagonal of the matrix for a healthy brain, and asymmetrical for pathological patients. Fig. 6 shows two examples of normal and abnormal MRI brain scanning and the corresponding co-occurrence matrix at angle $(0,0)$ of a normal and an abnormal brain scanning.

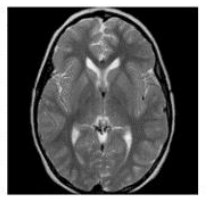

A

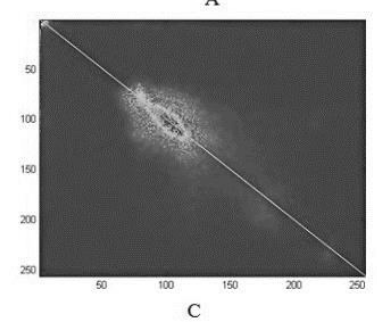

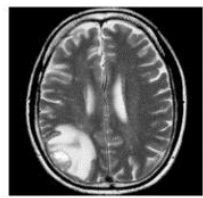

B

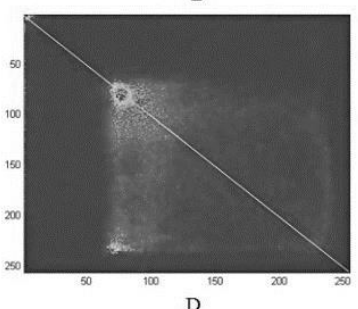

D

Figure 6: A) MRI normal brain scanning image, B) MRI abnormal brain scanning image,

C) MGLCM of normal brain scanning and D) MGLCM of abnormal brain scanning.

Finally, 19 co-occurrence statistics are extracted from each co-occurrence matrix using the expressions given in the Appendix representing the most common features derived from co-occurrence matrices [18, 19]. These will be used in addition to the weighted mean and weighted distance features that are proposed in this paper and explained in detail in the next paragraphs. These features were used to measure statistically the degree of symmetry between the two hemispheres of the brain because the 
symmetry represents the main indicator to detect pathological brains.

1-Weighted mean descriptor is proposed in this study to detect the irregularity of the MRI brain scanning image by calculating the nearest distance between the weighted mean to the diagonal of co-occurrence matrix as shown in Fig. 7.

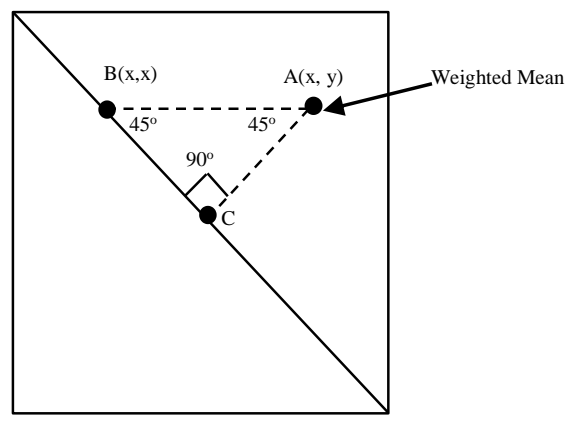

Figure 7: Weighted mean representation in co-occurrence matrix.

It is high when there is any abnormality in the MRI brain scanning image and low when there is normal brain scanning and defined using Equation 3 and Equation 4:

$$
\begin{aligned}
& x=\frac{1}{256^{2}} \sum_{i=1}^{256} \sum_{j=1}^{256} i . P(i, j) \\
& y=\frac{1}{256^{2}} \sum_{i=1}^{256} \sum_{j=1}^{256} j . P(i, j)
\end{aligned}
$$

Where $x$ and $y$ are the coordinates of weighted mean in the co-occurrence as given in Equation 5 .

$$
\text { Weighted mean }_{(A, B)}=|y-x| \sin 45^{\circ} \quad 5
$$

2-Weighted distance descriptor is also proposed in this study to detect the irregularity of the MRI brain scanning image by multiplying each coefficient in the co-occurrence matrix by the nearest distance $\boldsymbol{d}$ to the diagonal as shown in Fig. 8. It is high with abnormal brain scanning and low with normal brain scanning and defined using Equation 6 and Equation 7.

$$
u_{\text {trang. }}=\sum_{i} \sum_{j} d_{i j} \cdot P(i, j)
$$

Where, $i$ and $j$ are the element's coordinates of co-occurrence matrix that are located in the upper triangular of the matrix.

$$
\text { low }_{\text {trang. }}=\sum_{i} \sum_{j} d_{i j} \cdot P(i, j)
$$

Where, $i$ and $j$ are the element's coordinates of co-occurrence matrix that are located in the lower triangular of the matrix as shown in Equation 8.

$$
\text { Weighted destance }=\mid \text { up trang. }- \text { low trang. } \mid \quad 8
$$

Subsequently, there are 190 predictors that are extracted from 9 offsets for each MRI brain scanning.

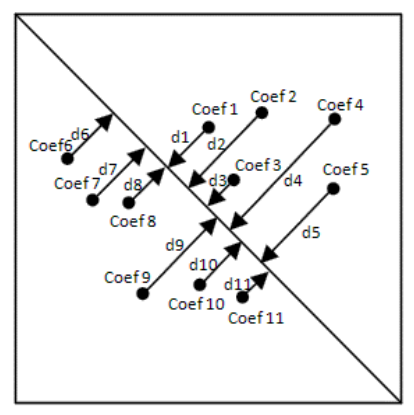


Figure 8: Weighted distance representation in MGLCM

\subsection{Preparing the Extracted Features for Classification}

There are some preprocessing steps that should be taken to prepare the extracted features for classification as they help in improving the accuracy, efficiency and scalability of the classification process [20]. Features Transformation is the process of transforming all extracted features by normalization, into new forms that are more appropriate for the classification process to prevent features with large ranges outweighing those from smaller ones. In this study, the min-max normalization approach is used to perform a linear transformation on the extracted features whilst preserving the relationships between the original features [20].

Relevance Analysis is the process of identifying and removing irrelevant and redundant features that do not contribute to the classification process and to reduce computational complexity by transforming high-dimensional data into a meaningful representation of the reduced dimensionality. It also contributes to improving training of the classifier to be faster, more effective and more accurate $[21,22]$. A redundant feature is defined as one that it is highly correlated with one or more of the other features, provided both of them are relevant, while the irrelevant predictor can be removed without affecting the classification performance. As stated by Hall [21], "a good feature subset is one that contains features that are highly correlated with the group and uncorrelated with each other".

In this study, stepwise analysis of variance ANOVA is used. It is a robust statistical technique used for data analysis and for detecting the level of significance of each predictor in the feature space based on calculating whether differences exist between two or more group means by analyzing the within-group and between-group variance. It predicts the significance of a predictor using F-statistic and P-values where the F-statistic is defined as the ratio of between-group variance to the within-group variance. The predictor that has the highest significant variation between classes has a high F-statistic value and a small P-value. From the ANOVA table, when the P-value for the F-statistic value is less than the critical value $\alpha$, then the predictor will be significant. The critical value $\alpha$ is proposed and fixed by Fisher [23]. He suggested the idea of a conventional probability for accepting or rejecting the hypothesis and it was one in twenty $(0.05$ or $5 \%)$. Consequently, the predictor will be significant when $\mathrm{P}<0.05$, very significant when $\mathrm{P}<0.01$, and highly significant when $\mathrm{P}<0.001$.

\section{Classification}

Classification is the process of sorting objects in an image into separated classes, and represents the final step of image processing. Three classifiers are applied and the results are compared. These classifiers are Linear Discriminant Analysis (LDA), Support Vector Machine (SVM), and Multi-layer Perceptron Neural Network (MLP). Training samples are randomly selected. A 10 -folded cross-validation is used to validate the robustness of our proposed system. Cross-validation also helps to prevent overfitting.

\section{Experimental Results}

To evaluate the proposed algorithms of this study a set of examples will be implemented using these algorithms.

\subsection{MSP Detection and Correction Results}

Fig. 9 shows the result of MSP detection and correction of the three MRI brain scanning images which are shown in different orientation in the first row. In the second row, the scans are corrected and aligned in the middle of image. The MRI image shown in Fig. 10, is re-sampled using the Geometric Rotator system object in the MATLAB Image Processing Toolkit [24], to rotate the patient's head with yaw angles from -10 to 10 degrees in 5 degree intervals. The proposed algorithm is evaluated by comparing our results with the proposed algorithms in [16] as shown in Table I. It is noted that there is a significant difference in the mean squared error (MSE) between both algorithms, and the performance of our algorithm outperforms those proposed in [16].

\subsection{Classification Results}

As indicated in section 4.2, 190 features were extracted for each image by the MGLCM method. Additionally, the LDA, SVM and MLP classifiers were used to classify the extracted texture features. The highest classification accuracy with best performance was achieved using the MLP network at $91 \%$ while LDA and SVM achieved $77 \%$ and $87 \%$ respectively. The same co-occurrence statistics which were used in this study were computed using the traditional grey level co-occurrence matrix (GLCM) method in order to perform a comparison between the two methods. Four co-occurrence matrices with four orientations $\left(0^{\circ}, 45^{\circ}, 90^{\circ}\right.$ and $\left.135^{\circ}\right)$ and distance 1 were computed. The maximum classification accuracy was achieved by MLP at $86 \%$, followed by $82 \%$ for SVM and the LDA classifier achieved $74 \%$. 


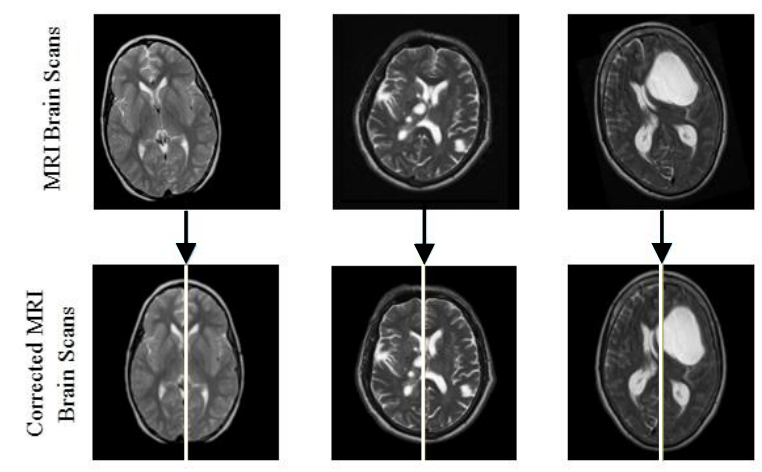

Figure 9: MSP detection and correction of three pathological patients.

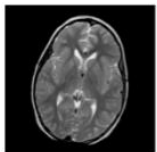

$-10$

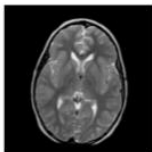

$-5$

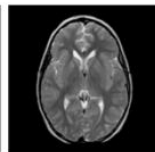

0

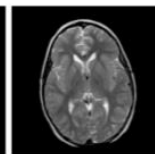

5

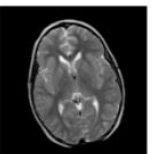

10

Figure 10: Re-sampling of one slice from the axial MRI brain scanning image with varied rotation angles.

TABLE I: NUMERICAL RESULTS OF DETECTING YAW ANGLE

\begin{tabular}{ccccccc}
\hline Yaw Angle & -10 & -5 & 0 & 5 & 10 & MSE \\
\hline Detected Yaw Angle & -9.1 & -4.7 & 0.5 & 5.4 & 10.5 & 0.3 \\
\hline$[16]$ & -8.5 & -3 & 1.25 & 6.5 & 11.2 & 2.87 \\
\hline
\end{tabular}

Additionally, the performance of the features which were extracted by MGLCM was compared with the Gabor wavelet features that were applied with five different scales and eight orientations using a window size of $(33 \times 33)$. The length of the Gabor feature vector was 655360 . The achieved classification accuracy by the three classifiers was $90 \%$ for SVM, $62.5 \%$ for LDA and $87.4 \%$ for MLP. As shown in Fig. 11, the classification accuracies of the texture features that were extracted using MGLCM are higher than those extracted using GLCM and Gabor wavelet features in all classifiers.

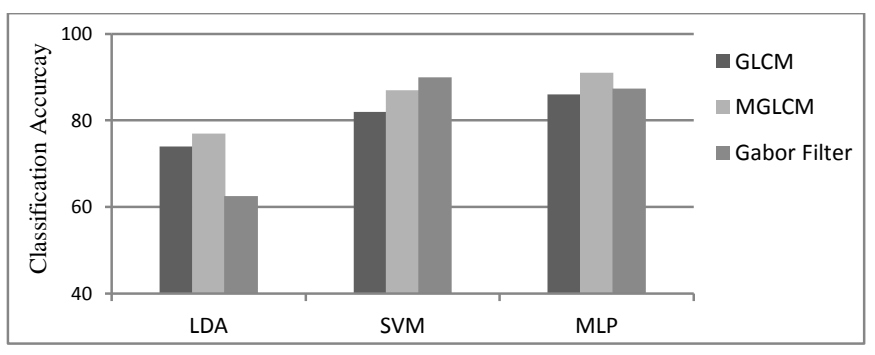

Figure 11: A comparison between MGLCM and GLCM classification accuracy.

After implementing the stepwise analysis of the variance ANOVA method for relevance analysis, in this study, the critical value $\alpha$ is set to 0.001 in order to obtain highly significant features. The assessment of the features will depend on both F-statistic value and P-value, as it is not enough that a predictor has a P-value that is less than 0.001 but it should also have a high Fstatistic value. Therefore a threshold value will be used to evaluate the significance of the predictors in addition to P-value. Once the F-statistic threshold value is set to zero, all predictors in the features vector will be selected. When the F-statistic threshold value is increased, the number of selected predictors decreases and the features vector is reduced. Therefore, to choose the optimal F-statistic threshold value, the classification accuracy of the three classifiers should be examined and evaluated. Different F-statistic threshold values are tested at each run when we measure the number of predictors which have an F-statistic 
value greater than the threshold. The results of the classification are shown in Table II.

TABLE II

THE RESULT OF USING FOUR DIFFERENT F-STATISTIC THRESHOLD VALUES ON THE CLASSIFICATION ACCURACY.

\begin{tabular}{cccccc}
\hline \multirow{2}{*}{$\begin{array}{c}\text { F-statistic } \\
\text { threshold value }\end{array}$} & $\begin{array}{c}\text { No. of selected } \\
\text { predictors }\end{array}$ & \multirow{2}{*}{$\begin{array}{c}\text { LDA } \\
\text { Accuracy }\end{array}$} & \multirow{2}{*}{$\begin{array}{c}\text { SVM } \\
\text { Accuracy }\end{array}$} & $\begin{array}{c}\text { Size of } \\
\text { hidden layer }\end{array}$ & Accuracy \\
\hline 30 & 127 & $81 \%$ & $95 \%$ & 60 & $96.6 \%$ \\
\hline 35 & 100 & $83 \%$ & $96 \%$ & 55 & $97.8 \%$ \\
\hline 50 & 55 & $82 \%$ & $95 \%$ & 25 & $94 \%$ \\
\hline
\end{tabular}

Fig. 12 shows that the classification accuracy of the three classifiers has improved and reached the maximum value when the F-statistic threshold value is set to 35 . It also shows that the average performance of LDA and SVM models were $83 \%$ and $96 \%$ respectively. The transfer function used in the MLP network was the tangent function and the training function. To update the weights and bias values, the scaled conjugate gradient method (trainscg) that is suggested by MATLAB to be faster than the default function (trainlm) for larger datasets, is used. The training of the MLP network with 100 input layer neurons, 55 hidden layer neurons and single output layer is shown in Fig. 13. The average performance of the MLP network was $97.8 \pm 0.1 \%$ and sensitivity and specificity rates were $98.1 \pm 0.3 \%$ and $97.6 \pm 0.4 \%$ respectively. Additionally, there was just one non-pathological patient that was classified incorrectly as pathological and three pathological patients that were classified incorrectly as nonpathological.

Subsequently, the number of predictors in the feature vector is reduced from 190 to 100 predictors with 90 predictors discarded and considered as irrelevant or redundant features. Eleven relevant and significant features for each angle of the MGLCM are chosen by the ANOVA method namely: contrast, correlation, dissimilarity, sum of square variance, sum average, sum variance, difference entropy, information measure of correlation 1, inverse difference normalized (IDN), inverse difference moment normalized (IDMN) and weighted distance in addition to the cross correlation.

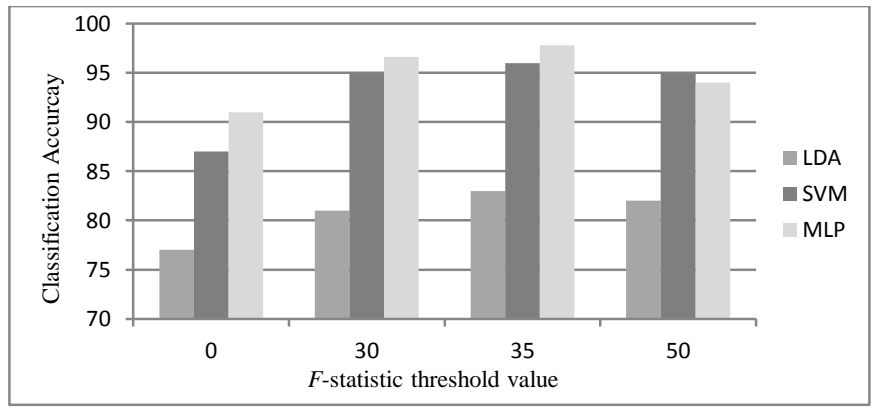

Figure 12: The optimal F-statistic threshold value.

The ANOVA method was also applied on the texture features which were extracted by the traditional GLCM. The best classification accuracy was achieved when the F-statistic threshold value was 35 , such that the selected texture features were auto correlation, cluster prominence, cluster shade, sum of square variance, sum variance and cross correlation. The maximum classification accuracy of MLP, SVM and LDA were 92\%, 90\% and 79\% respectively. It can be clearly seen that the classification accuracy of the used classifiers were significantly higher when using MGLCM method than GLCM as shown in Fig. 14.

The performance of ANOVA is validated by comparing its results against those obtained by other techniques for feature selection mainly PCA and Kernel PCA methods [22, 25]. Table III and Table IV show the results when using PCA and Kernel PCA respectively as feature selection and reduction methods with the same dimensions that were used with ANOVA (127, 100 and 55) and the results of classification accuracy of the same classifiers that were used previously in this study. 


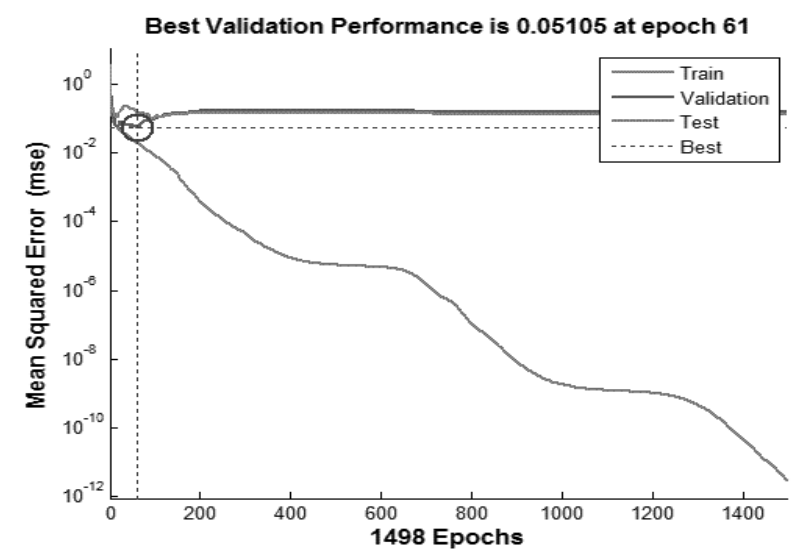

A

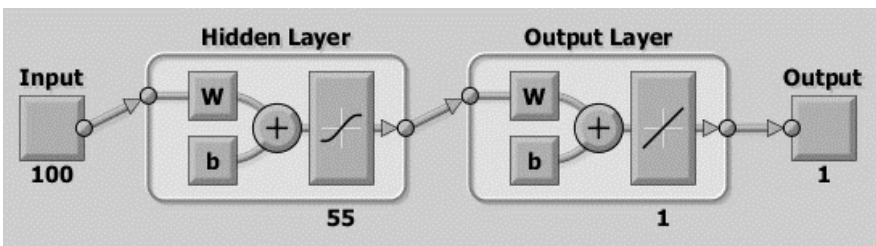

$\mathrm{B}$

Figure 13: A) MLP network structure and B) The performance of MLP network.

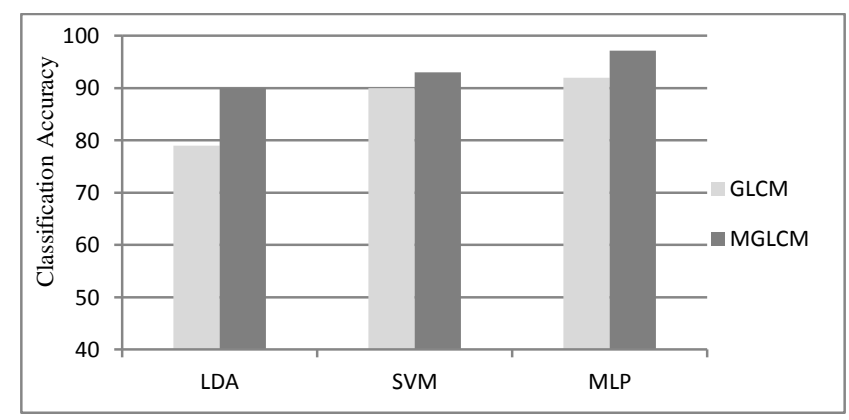

Figure 14: A comparison between MGLCM and GLCM regard classification accuracy when using ANOVA method.

TABLE III

CLASSIFICATION ACCURACY FOR THE THREE CLASSIFIERS WITH PCA METHOD FOR FEATURE SELECTION.

\begin{tabular}{ccccc}
\hline \multirow{2}{*}{$\begin{array}{c}\text { No. of selected } \\
\text { predictors }\end{array}$} & LDA & SVM & \multicolumn{2}{c}{ MLP } \\
\cline { 4 - 5 } & Accuracy & Accuracy & $\begin{array}{c}\text { Size of hidden } \\
\text { layer }\end{array}$ & Accuracy \\
\hline 127 & $65 \%$ & $78 \%$ & 65 & $66 \%$ \\
\hline 100 & $63 \%$ & $80 \%$ & 55 & $77 \%$ \\
\hline 55 & $63 \%$ & $78 \%$ & 25 & $78 \%$ \\
\hline
\end{tabular}

TABLE IV

CLASSIFICATION ACCURACY FOR THE THREE CLASSIFIERS WITH KERNEL PCA METHOD FOR FEATURE SELECTION.

\begin{tabular}{ccccc}
\hline No. of selected & LDA & SVM & \multicolumn{2}{c}{ MLP } \\
\cline { 4 - 5 } predictors & Accuracy & Accuracy & Size of hidden layer & Accuracy \\
\hline 127 & $60 \%$ & $81 \%$ & 65 & $62 \%$ \\
\hline 100 & $61 \%$ & $74.5 \%$ & 55 & $67 \%$ \\
\hline 55 & $58 \%$ & $76 \%$ & 25 & $72 \%$ \\
\hline
\end{tabular}


The Correlation Feature Selection Subset Evaluator (CFS-SE) method is also used for the evaluation and validation of the extracted features. This method is implemented using the WEKA software [9]. In total, six predictors for the entire angles are chosen as the most relevant by this method and they are weighted distance $(0,0)$, sum of square variance $(0,315)$, dissimilarity $(315,45)$, inverse difference normalize $(315,45)$, inverse difference normalize $(315,315)$ and inverse difference moment normalize (45,315). Table V summarizes the result of the classification of the selected features using the WEKA CFS-SE method with the same classifiers that were used previously.

TABLE V

CLASSIFICATION ACCURACY FOR THE THREE CLASSIFIERS WITH WEKA CFS-SE METHOD FOR FEATURE SELECTION.

\begin{tabular}{ccccc}
\hline No. of selected & LDA & SVM & \multicolumn{2}{c}{ MLP } \\
\cline { 4 - 5 } predictors & Accuracy & Accuracy & Size of hidden layer & Accuracy \\
\hline 6 & $75 \%$ & $89 \%$ & 3 & $91 \%$ \\
\hline
\end{tabular}

Fig. 15 summarizes the behavior of the implemented features selection methods and shows that the behaviour of ANOVA outweighs the performance of the other techniques for detecting the most relevant predictors.

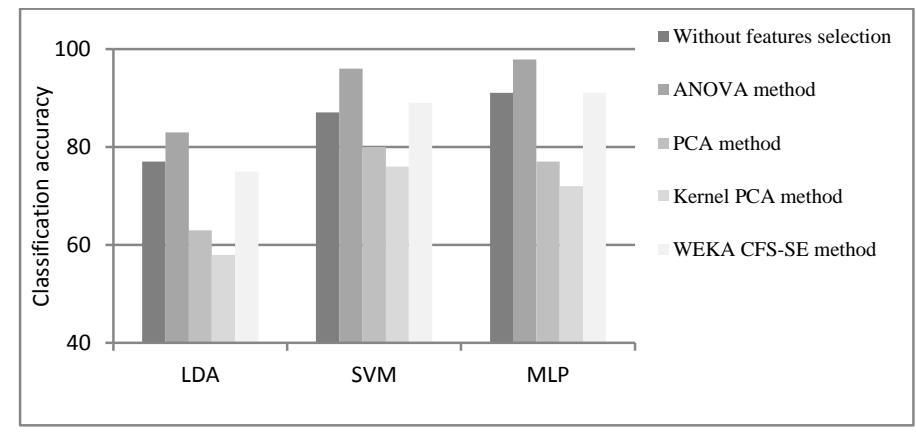

Figure 15: Performance of the implemented methods for feature selection.

\section{Discussion}

Since visual diagnosis of the MRI scans is subjective and depends on the expertise of the radiologist, texture analysis has been widely studied for improving the diagnosis of MRI brain scans. In this study, 19 co-occurrence statistics which are most popular and common in previous studies, in addition to two additional texture features which were proposed in this study, were extracted from nine MGLCM matrices to discriminate brain abnormalities. Only 11 co-occurrence statistics in addition to cross correlation predictor were chosen as the most significant features by ANOVA. The weighted distance feature proposed in this study, was included within these 11 co-occurrence statistics and was chosen by ANOVA as a significant feature. The experimental results of the proposed algorithm are compared with previous studies as shown in Table VI.

TABLE VI

COMPARISON WITH PREVIOUS PROPOSED METHODS

\begin{tabular}{|c|c|c|c|c|}
\hline Reference & Features methods & No. of Patients & Classifier & Accuracy \\
\hline [10] & $\begin{array}{l}\text { - First-order statistical } \\
\text { - GLCM (4 orientations and } 2 \text { distances) } \\
\text { - GLRLM (4 orientations) } \\
\text { - HOG } \\
\text { - LBP }\end{array}$ & 25 & SVM & $97.4 \%$ \\
\hline [14] & -Searching about pathological area by symmetry checking & 203 & SVM & $91.15 \%$ \\
\hline [19] & - GLCM (4 orientations and 10 distances) & 436 & LDA & $87 \%$ \\
\hline $\begin{array}{l}\text { Proposed } \\
\text { System }\end{array}$ & - MGLCM (9 orientations and 1 distance) & 165 & MLP & $97.8 \%$ \\
\hline
\end{tabular}

The authors in [10] used five methods for feature extraction; first-order statistical features, GLCM with four orientations $\left(0^{\circ}\right.$, $45^{\circ}, 90^{\circ}$ and $\left.135^{\circ}\right)$ and two distances ( 1 and 2), GLRLM with four orientations $\left(0^{\circ}, 45^{\circ}, 90^{\circ}\right.$ and $\left.135^{\circ}\right)$, a histogram of oriented gradient features HOG and linear binary pattern features LBP. The feature vector included 475 predictors. The dataset included 25 patients. A classification accuracy of $97.4 \%$ was achieved by SVM. If we consider that there were 5 feature extraction methods, and the processing time of first-order statistical method, HOG and LBP was 3n, the GLCM required 8n because there 
were four orientations and two distances and the GLRLM required $4 \mathrm{n}$ because there were four orientations. Then, the total processing time is $\mathrm{TP}=15 \mathrm{n}$. By using the big-O notation, we could write $\mathrm{Tf}=\mathrm{O}(15 \mathrm{n})$, which indicates that the complexity of the previous algorithm depends mainly on the number of feature extraction methods.

The authors in [19] used the GLCM method for feature extraction to classify breast ultrasound images (BUS) with 22 predictors computed from four co-occurrence matrices with orientations $\left(0^{\circ}, 45^{\circ}, 90^{\circ}\right.$ and $\left.135^{\circ}\right)$, ten distances $(1-10$ pixels $)$ and six quantization levels $(8,16,32,64,128$ and 256). To reduce the dimensionality of the feature space, the texture descriptors of the same distance were averaged over all orientations from 880 to 220 . Additionally, mutual information (MI) is used for evaluating the quality of the features subset. The maximum classification accuracy was achieved by the LDA classifier at $87 \%$ for classifying 436 BUS images. The selected predictors were feature/ $\theta / \mathrm{d}$; correlation I/90/8, cluster prominence/0/1, correlation II/90/8, contrast/90/1, correlation I/90/9, difference variance/90/1, correlation II/90/9, correlation I/90/2, correlation I/90/7, inverse difference moment normalize/90/1, correlation II/90/7, correlation I/90/10, correlation I/90/6, correlation II/90/10, correlation II/90/6, correlation I/90/5 and inverse difference moment normalize/90/1. The total processing time is TP=9n and using the big-O notation, we could write $\mathrm{Tf}=\mathrm{O}(9 \mathrm{n})$.

The automated screening algorithm in this study depends essentially on the single proposed method for texture feature extraction MGLCM with nine orientations. The significant features were selected using the ANOVA method and were reduced to 100 predictors. Over the entire dataset which included 165 patients, the average achievable accuracy was $97.8 \%$ by using MLP. The big-O notation of the proposed algorithm is $\mathrm{Tf}=\mathrm{O}(9 \mathrm{n})$.

\section{Conclusion}

An automated screening algorithm of MRI brain scanning images is developed to identify brain abnormality. This algorithm helps clinicians to improve the accuracy of the diagnosis and to reduce the diagnosing time. It is observed that the statistical texture features which were extracted by MGLCM are sufficient to discriminate the pathological patients from non-pathological patients by using T2 weighted MRI images because most of the brain tumors appear hyperintense in these images relative to normal brain tissue. It is also noted, that the achieved accuracy with low computational complexity demonstrates the efficiency of the proposed method for features extraction and its independence from the atlas registration method. A further advantage of our approach is that it uses a single MRI scan modality (T2 weighted image). The MGLCM gives high performance and accuracy in discriminating the normality and abnormality of the brain. However, the method is computationally expensive and memory requirements represent disadvantage.

\section{Acknowledgement}

We would like to thank the reviewers for their insightful comments on the paper, as these comments led to improve the quality of the paper and the MRI Unit of Al Kadhimiya Teaching Hospital in IRAQ for providing us with the MRI brain scanning images dataset.

\section{Appendix}

The 19 texture features used in our work were extracted from the MGLCM, these features are summarized below [19]:

$$
\begin{aligned}
& \text { Autocorrelationpredictor }=\sum_{i=0}^{N} \sum_{j=0}^{N}(i . j) P(i, j) \\
& \text { Contrast }=\sum_{n=0}^{n-1} n^{2}\left\{\sum_{i=0}^{N} \sum_{j=0}^{N} P(i, j)\right\} \text { wheren }=\mid i-j \\
& \text { Correlation }=\sum_{i=0}^{N-1} \sum_{j=0}^{N-1} P(i, j) \frac{\left(i-\mu_{x}\right)\left(j-\mu_{y}\right)}{\sigma_{x} \sigma_{y}} \\
& \text { Entropy }-\sum_{i=0}^{N-1} \sum_{j=0}^{N-1} P(i, j) \log P(i, j) \\
& \text { Energy }=\sum_{i=0}^{N-1} \sum_{j=0}^{N-1} P(i, j)^{2}
\end{aligned}
$$




$$
\begin{aligned}
& \text { Homogeneity }=\sum_{i=0}^{N-1} \sum_{j=0}^{N-1} \frac{1}{1+(i-j)^{2}} \cdot P(i, j) \\
& \text { Dissimilarity }=\sum_{i=0}^{N-1} \sum_{j=0}^{N-1}|i-j| P(i, j) \\
& \text { Sumofsquarevariance }=\sum_{i=0}^{N-1} \sum_{j=0}^{N-1}(i-\mu)^{2} \cdot P(i, j) \\
& \text { Clustershade }=\sum_{i=0}^{N-1} \sum_{j=0}^{N-1}\left(i+j-\mu_{x}-\mu_{y}\right)^{3} P(i, j) \\
& \text { Clusterprominence }=\sum_{i=0}^{N-1} \sum_{j=0}^{N-1}\left(i+j-\mu_{x}-\mu_{y}\right)^{4} P(i, j) \\
& \text { Inversedifferencenormalized }=\sum_{i=0}^{N-1} \sum_{j=0}^{N-1} \frac{1}{1+\frac{|i-j|}{N}} P(i, j) \\
& \text { Inversedifferencemomentnormalized }=\sum_{i=0}^{N-1} \sum_{j=0}^{N-1} \frac{P(i, j)}{1+\frac{(i-j)^{2}}{N}} \quad 12 \\
& \text { Sumaveragepredictor }=\sum_{i=1}^{2 N-1} i P_{i+j}(i, j) \\
& \text { Sumenropypredictor }=-\sum_{i=1}^{2 N-1} P_{i+j}(i, j) \log P_{i+j}(i, j) \\
& \text { Sumvariancepredictor }=\sum_{i=1}^{2 N-1}(i-\text { sumentropy })^{2} P_{i+j}(i, j) \\
& \text { Differenceenropypredictor }=-\sum_{i=0}^{N-1} P_{i-j}(i, j) \log P_{i-j}(i, j) \\
& \text { Informationmeasureof correlationI }=\frac{H X Y-H X Y 1}{\max (H X, H Y)} \\
& \text { Informationmeasureof correlationII }=\sqrt{1-e^{(-2(H X Y 2-H X Y))}} \quad 18 \\
& \text { Maximumprobabilitypredictor }=\max _{i, j} P(i, j)
\end{aligned}
$$

\section{References}

[1] N. Nabizadeh, "Automated Brain Lesion Detection and Segmentation Using Magnetic Resonance Images," PhD Thesis, Department of Electrical and Computer Engineering, University of Miami, USA, 2015.

[2] B. Saha, N. Ray, R. Greiner, A. Murtha, and H. Zhang, "Quick detection of brain tumors and edemas: A bounding box method using symmetry", Computerized Medical Imaging and Graphics, vol. 36, No 2, pp. 95-107, 2012.

[3] D. Mortazavi, A. Z. Kouzani, and H. Soltanian-Zadeh, "Segmentation of multiple sclerosis lesions in MR images: a review", Neuroradiology, vol. 54, No 4, pp. 299-320, 2012.

[4] B. H. Menze, A. Jakab, S. Bauer, J. Kalpathy-Cramer, K. Farahani, J. Kirby, et al., "The Multimodal Brain Tumor Image Segmentation Benchmark (BRATS)", IEEE Transactions on Medical Imaging, vol. 34, Issue 10, pp. 1993-2024, 2015.

[5] R. Fathi, L. Matti, H. Al-Salih, and D. Godbold, "Environmental pollution by depleted uranium in Iraq with special reference to Mosul and possible effects on cancer and birth defect rates," Medicine, Conflict and Survival, vol. 29, No 1, pp. 7-25, 2013.

[6] A. Kharrat, G. Karim, M. Ben Messaoud, N. Benamrane, and M. Abid, "A Hybrid Approach for Automatic Classification of Brain MRI Using Genetic Algorithm and Support Vector Machine," Leonardo Journal of Sciences, issue 17,. pp. 71-82, 2010.

[7] G. Pantelis, "Computer Assisted Diagnosis of Brain Tumors based on Statistical Methods and Pattern Recognition Techniques," PhD Thesis, Interdepartmental Postgraduate Program in Medical Physics, University of Patras, 2010.

[8] S. Lahmiri and M. Boukadoum, "Hybrid Discrete Wavelet Transform and Gabor Filter Banks Processing for Features 
Extraction from Biomedical Images," Journal of Medical Engineering, vol. 2013, pp. 1-13, 2013.

[9] J. Sachdeva, V. Kumar, I. Gupta, N. Khandelwal, and C. Ahuja, "Segmentation, Feature Extraction, and Multiclass Brain Tumor Classification," Swetswise, vol. 26, No 6, pp. 1141-1150, 2013.

[10] N. Nabizadeh and M. Kubat, "Brain tumors detection and segmentation in MR images: Gabor wavelet vs. statistical features," Computers \& Electrical Engineering, vol. 45, pp. 286-301, 2015.

[11] G. Ruppert, L. Teverovskiy, Y. Chen-Ping, X. Falcao, and L. Yanxi, "A new symmetry-based method for mid-sagittal plane extraction in neuroimages", International Symposium on Biomedical Imaging: From Nano to Macro, Chicago, IL, 2011, pp. 285-288.

[12] S. Jayasuriya and A. Liew, "Symmetry plane detection in neuroimages based on intensity profile analysis", The International Symposium on Information Technology in Medicine and Education (ITME), pp.599-603, Australia 2012.

[13] U. Stańczyk and L. Jain, Feature Selection for Data and Pattern Recognition: An Introduction. In U. Stańczyk and L. Jain (Eds), Feature Selection for Data and Pattern Recognition, Springer Series on Studies in Computational Intelligence vol. 584, pp: 1-7, Berlin Heidelberg: Springer, 2015.

[14] P. Dvořák, W. Kropatsch, and K. Bartušek, "Automatic brain tumor detection in t2-weighted magnetic resonance images," Measurement Science Review, vol. 13, No 5, pp. 223-230, 2013.

[15] S. Tantisatirapong, "Texture analysis of multimodal magnetic resonance images in support of diagnostic classification of childhood brain tumours," $\mathrm{PhD}$ Thesis, School of Electronic, Electrical and Computer Engineering, University of Birmingham, 2015.

[16] Y. Liu and R. Collins, "Automatic Extraction of the Central Symmetry (MidSagittal) Plane from Neuroradiology Images," Technical report, CMU-RI-TR-96-40, Robotics Institute, Carnegie Mellon University,1996.

[17] M. Sonka, V. Hlavac, and R. Boyle, Image Processing, Analysis, ans Machine Vision, third edition ed., 2008.

[18] M. Haralick, K. Shanmugam and I. H. Dinstein, "Textural Features for Image Classification,", IEEE Transactions on Systems, Man and Cybernetics, vol. SMC-3, Issue 6, pp. 610-621, 1973.

[19] W. Gomez, W. Pereira, and A. Infantosi, "Analysis of Co-Occurrence Texture Statistics as a Function of Gray-Level Quantization for Classifying Breast Ultrasound", IEEE Transactions on Medical Imaging, vol. 31, issue 10, pp. 18891899, 2012.

[20] J. Han and M. Kamber, Data Mining: Concepts and Techniques. USA: Diane Cerra, 2006.

[21] M. Hall, "Correlation-based Feature Selection for Machine Learning," PhD Thesis, Department of Computer Science, University of Waikato, NewZealand, 1999.

[22] P. Van der Maaten, "An Introduction to Dimensionality Reduction Using Matlab," Technical Report, Report MICC 0707 , Faculty of Humanities \& Sciences, Maastricht University, The Netherlands, 2007.

[23] G. Quinn and M. Keough, Experimental Design and Data Analysis for Biologists, Cambridge Press, UK, 2002.

[24] Matlab, "The Math Works kit," ed, 2013.

[25] Matlab Toolbox for Dimensionality Reduction. (2015). Available: http://lvdmaaten.github.io/drtoolbox/

Ali. M. Hasan is currently a Ph.D. candidate in the School of Computing, Science and Engineering at the University of Salford, UK. Prior to his PhD studies, he was a lecturer in the College of Medicine at Al-Nahrain University, Iraq. He can be contacted at: ali11alwaeli@yahoo.com; a.hasan4@edu.salford.ac.uk.

Farid Meziane is a professor in Data and Knowledge Engineering in the School of Computing, Science and Engineering at the University of Salford, UK. He is the director of the Informatics Research Centre. He can be contacted at: f.meziane@salford.ac.uk. 